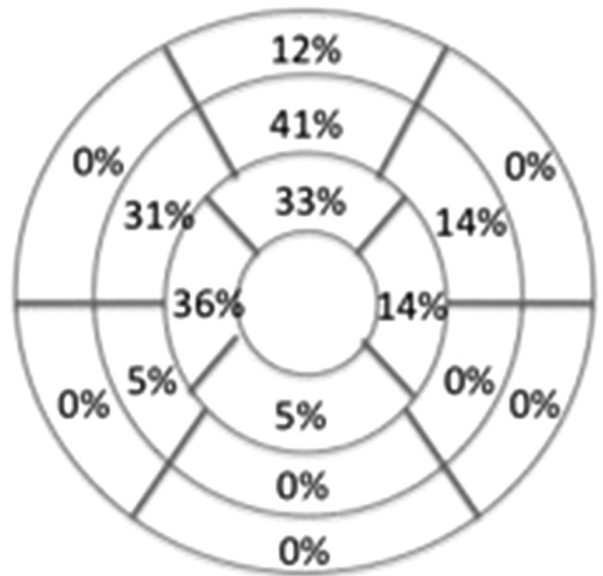

Cine sequences

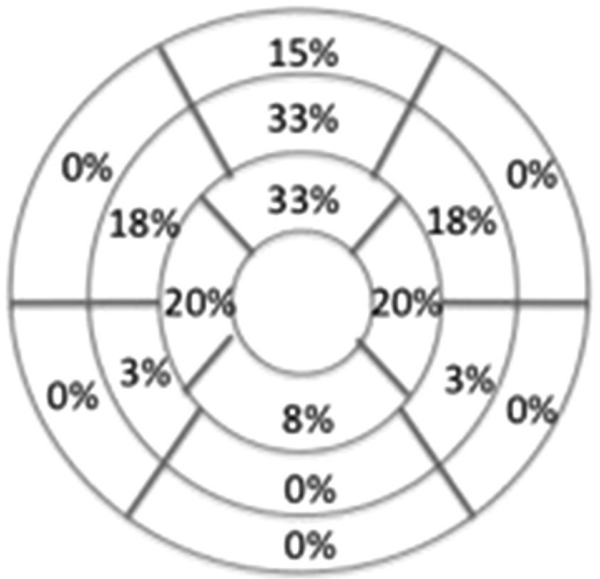

Post-contrast sequences

Abstract 2 Figure 1 Left ventricular segmental analysis to assess artefacts interference. Sixteen-segment model showing that artefacts mostly affect the mid-apical left ventricular anterior and anteroseptal walls, on cine and post-contrast sequences, respectively

(kappa 0.284, $\mathrm{p}=0.005$ ) (Table 1). Cine sequences were most affected by artefacts, mainly in the mid-apical left ventricular anterior wall and anteroseptum (Figure 1). No change in device parameters was reported after the scan. CMR had a clinical impact in 26 patients (57\%), determining a change in diagnosis in $16(35 \%)$, in management in $5(11 \%)$ and a change in both in 5 patients $(11 \%)$.

Conclusion With dedicated protocols and under strict monitoring of cardiac devices, CMR is safe and feasible in patients wearing MR-conditional devices, and it also has major clinical impact.

\section{CLINICAL UTILITY OF CARDIAC MRI IN YOUNG-MIDDLE AGED PATIENTS WITH HIGH-GRADE ATRIO- VENTRICULAR BLOCK}

${ }^{*}$ A Baritussio, A Ghosh Dastidar, N Ahmed, J Rodrigues, A Frontera, C Lawton, D Augustine, E McAlindon, C Bucciarelli-Ducci. NIHR Cardiovascular Biomedical Research Unit, Bristol Heart Institute, Bristol, UK

\subsection{6/heartjnl-2016-309668.3}

Background Atrio-ventricular (AV) block is a rare event in young-middle aged adults, often leading to pacemaker implantation without further investigation. We sought to assess the clinical utility of CMR in young-middle aged adults with high-grade AV block.

Methods We retrospectively analysed the CMR registry to collect data on consecutive high-grade AV block patients (18-60yrs) referred for CMR (September 2012-November 2015). Highgrade AVB was defined as Mobitz II $2^{\text {nd }}$ degree or complete AVB. All patients underwent a transthoracic echocardiogram (TTE) and a comprehensive CMR protocol (cine and late gadolinium enhancement, LGE). A change in diagnosis was defined as a new diagnosis compared to a multi-parametric pre-CMR diagnosis (based on clinical, ECG and TTE data).

Results We identified 34 patients (20 male, mean age $44 \pm 12$ years); 12 patients (34\%) had II degree AVB and 22 (66\%) complete AVB. Patients were referred to CMR for suspected ischaemic heart disease (IHD) in 4 patients (11\%) and non ischaemic heart disease (NIHD) in $24(71 \%)$; in 6 patients $(18 \%)$ preCMR diagnosis was unclear. CMR showed IHD in 3 patients (9\%) and NIHD in 11 patients (32\%); a structurally normal heart was found in 18 patients (53\%) and non-specific findings in $2(6 \%)$ (Table 1) (Figure 1). LGE was found in 12 patients (34\%), with predominant mid-wall pattern (58\%). There was

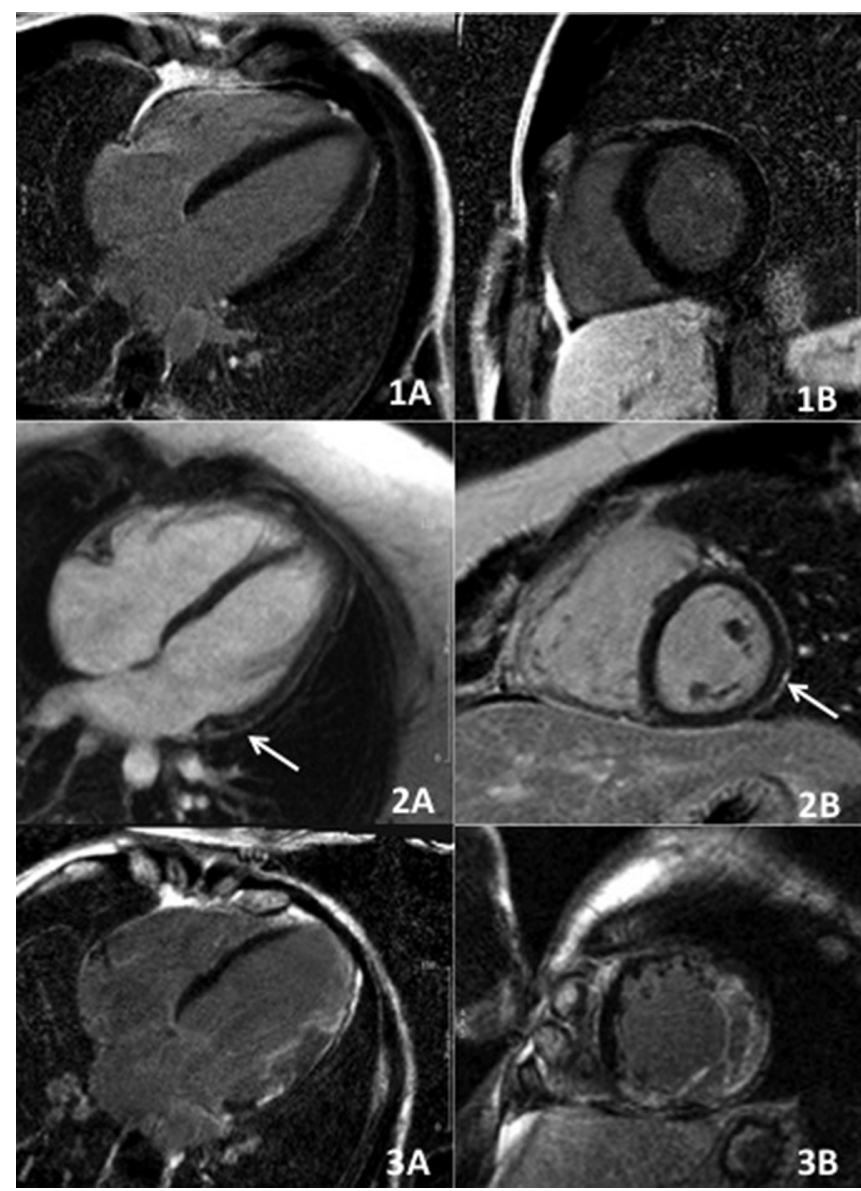

Abstract 3 Figure 1 CMR findings. Post-contrast four chamber longaxis $(1 \mathrm{~A})$ and short-axis (1B) view showing structurally normal heart. Post-contrast four chamber long-axis (2A) and short-axis (2B) view showing epicardial LGE in the basal to mid-cavity lateral wall (white arrow) in a patient with myocarditis. Post-contrast four chamber longaxis (3A) and short-axis (3B) view showing transmural myocardial LGE in the basal to apical lateral wall in a patient with left ventricular non compaction 
moderate agreement between CMR and TTE final diagnosis (Cohen's kappa 0.435, p 0.001). CMR determined a change in diagnosis in 14 patients (40\%).

Conclusions CMR was diagnostic in $94 \%$ of young-middle aged patients presenting with high grade AVB. As compared to a multi-parametric pre-CMR diagnosis, CMR led to a change in diagnosis in $40 \%$ of patients.

\section{Abstract 3 Table 1 CMR diagnosis}

\begin{tabular}{ll} 
CMR diagnosis & $\mathbf{n}=\mathbf{3 4}$ \\
\hline Ischaemic Heart Disease, $\mathrm{n}(\%)$ & $3(9)$ \\
Non-ischaemic Heart Disease, $\mathrm{n}(\%)$ & $11(32)$ \\
Structurally Normal Heart, $\mathrm{n}(\%)$ & $18(53)$ \\
Non-specific Findings, $\mathrm{n}(\%)$ & 2 (6) \\
\hline
\end{tabular}

4 TROPONIN POSITIVE ACUTE CORONARY SYNDROMES AND UNOBSTRUCTED CORONARY ARTERIES: IMPROVED DIAGNOSTIC AND CLINICAL IMPACT BY PERFORMING CARDIOVASCULAR MAGNETIC RESONANCE EARLY AFTER PRESENTATION

${ }^{1,2, *}$ A Ghosh Dastidar, ${ }^{1,3}$ J Rodrigues, ${ }^{1,2} \mathrm{~T}$ Johnson, ${ }^{1} \mathrm{E}$ De Garate, ${ }^{2} \mathrm{P}$ Singhal, ${ }^{1} \mathrm{~A}$ Baritussio, ${ }^{1} \mathrm{~A}$ Scatteia, ${ }^{1} \mathrm{~J}$ Strange, ${ }^{1,2} \mathrm{~A}$ Nightingale, ${ }^{1,2} \mathrm{~A}$ Baumbach, ${ }^{4} \mathrm{~V}$ Delgado, ${ }^{1,2} \mathrm{C}$ Bucciarelli-Ducci. ${ }^{1}$ NIHR Bristol Cardiovascular Biomedical Research Unit, Bristol Heart Institute, UK; ${ }^{2}$ School of Clinical Sciences, Faculty of Health Sciences, University of Bristol, UK; ${ }^{3}$ School of Physiology, Pharmacology and Neuroscience, Faculty of Biomedical Sciences, University of Bristol University of Bristol, UK; ${ }^{4}$ Department of Cardiology, Leiden University Medical Center, Leiden, The Netherlands

10.1136/heartjnl-2016-309668.4
Background $7-15 \%$ of acute coronary syndrome (ACS) patients have unobstructed coronary arteries. In these patients cardiac magnetic resonance (CMR) can identify different underlying aetiologies.

Aim Evaluate the diagnostic and decision making implications of CMR timing (early versus late) in patients with ACS and unobstructed coronary arteries.

Methods 204 consecutive patients (mean age 55yrs, 51\% males) with troponin positive ACS and unobstructed coronary arteries, referred for a CMR between September 2011 and July 2014 were evaluated. Comprehensive CMR was performed "early" ( $\leq 2$ weeks from presentation) in 98 patients and "late" (>2weeks from presentation) in 106. "Significant clinical impact" was predefined as change in diagnosis/management. Propensity matching was performed between early and late CMR groups to minimise selection bias.

Results Overall, a cause was found in $70 \%$ of patients. CMR had significant clinical impact in $66 \%$, including change in the final diagnosis in 54\%. (Figure 1) In a multivariable model (included clinical and imaging parameters), presence of late gadolinium enhancement (LGE) and age were the only independent predictors of "significant clinical impact" (LGE OR 2.3, $\mathrm{p}=0.02$ ) (Table 1 ). In a propensity score analysis, 58 pair of patients was matched for early and late CMR. The diagnostic pick up rate in the "early" group was significantly higher than in the "late" group (88\% vs 50\% p < 0.0001). Myocarditis (33\%) was the most common diagnosis in the "early" group, whereas myocardial infarction (22\%) in the "late" group. The clinical impact also improved significantly in the early group compared to the propensity score matched late group (76\% vs $51 \%$, p = $0.01)$.

Conclusion CMR was able to establish final diagnosis in overall $70 \%$. CMR made significant additive clinical impact on management and diagnosis in 66\%, with LGE being the best

\section{Change in diagnosis after CMR}

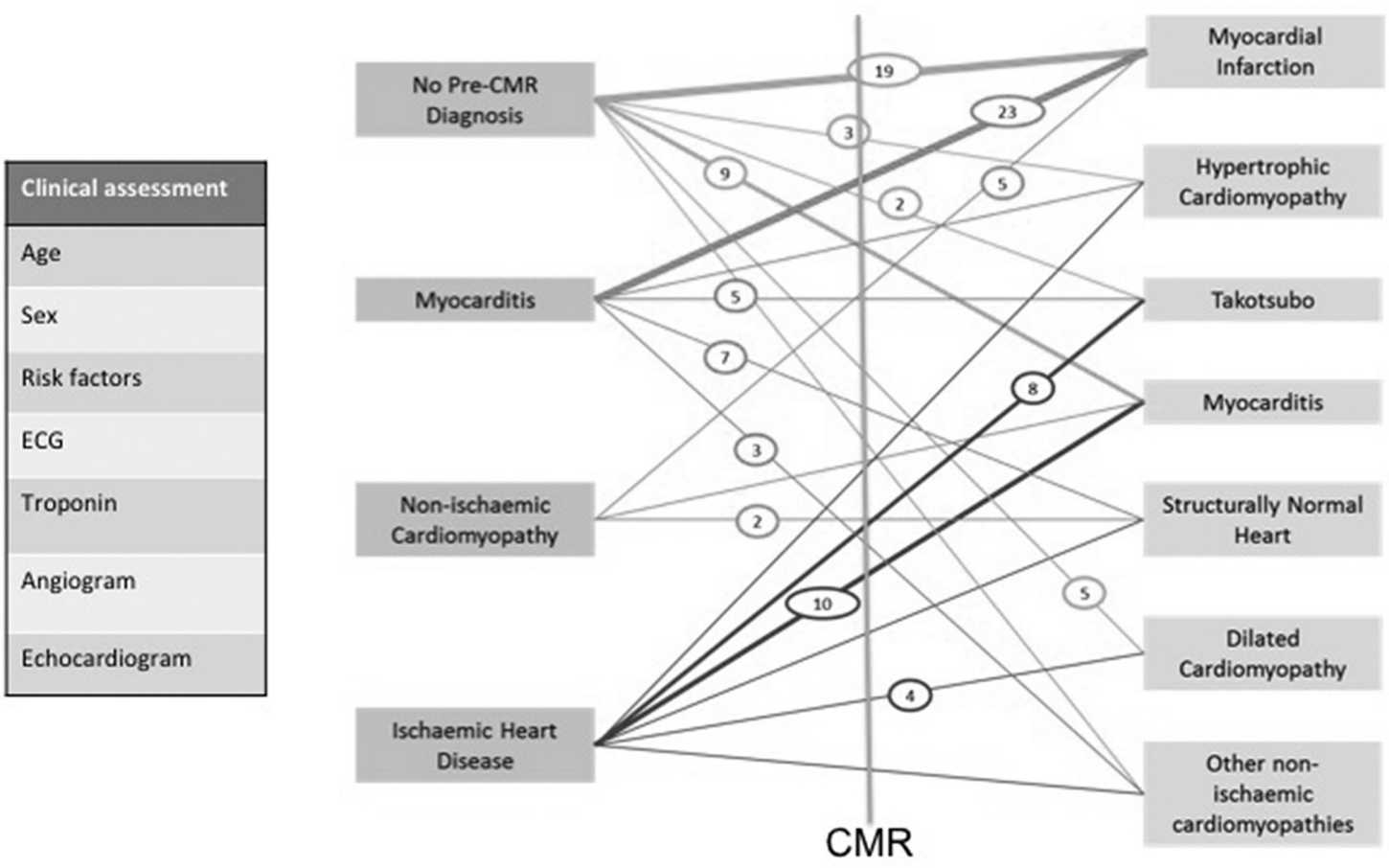

Abstract 4 Figure 1 Showing the change in diagnosis following CMR 